\title{
The Polarization Properties of Horn Antenna
}

\author{
Bingxia Cao, Jinghong Xue \\ School of Information and Electrical Engineering \\ Harbin Institute of Technology, HIT \\ Weihai, China \\ jchdd1980@163.com, xuejing_h@126.com
}

\author{
Jingjing Zhang \\ Shandong Astronautics Electronic Technology Institute \\ Yantai, China \\ Summer2002198320@163.com
}

\begin{abstract}
Polarization properties of horn antenna are studied in the paper. The linear and circular polarized horns are simulated. There are four lobes in the cross-polarization pattern of linear polarized horn. And the phase of cross-polarization is changed at each bound of the quadrants. That of co-polarization, meanwhile, keeps almost the same value. There is a notch in the center of circular polarized horn cross polarization pattern. And the phase of both co-polarization and cross-polarization is continuous. Far field and near field measurement of horn antenna is analyzed to demonstrate the simulation.
\end{abstract}

Keywords—polarization; horn; cross-polarization; phase

\section{INTRODUCTION}

There are two types of technical measure for the enhancement of anti- active jamming in radar system according to radar signal processing $[1,2]$. One can be called space signal processing method[3,4]. It means that the electromagnetic waves should be processed before entering the receiver. The interfering electromagnetic waves are undermined or reduced and the useful signal voltage level is promoted to improve the quality of signal reception. The other is the common signal processing $[5,6]$. The receiver firstly amplify and filter the waves. Appropriate signal processing is carried out for the waves according to the differences on wave forms, spectrum structure or the polarization between the useful signal and jamming for suppression of interference.

In the field of radar anti-active jamming in polarization, polarization filtering and polarization discrimination of active decoy are the main anti-interference methods[7-10].Both methods are based on the difference between the active jamming and radar target in polarization characteristics. So the polarization characteristics of the antenna should be studied firstly.

Horn antennas are widely used as feeds for reflector antennas[11], as elements for phased arrays[12, 13] and as reference antennas for testing and evaluation purposed[14, 15]. Corrugated horn antennas are one class of antennas that can achieve very low cross-polarization levels[15, 16]. The highly symmetric field distribution at the aperture contributes to the low cross polarization property.

In this paper, horn antenna of both linear and circular polarized are simulated to study the polarization characteristics. And also some measurement data of horn antenna to analyze the polarization properties.

\section{DEFINITION OF CROSS POLARIZATION}

Early in the 1970s, the study on the polarization characteristics of antenna was reported[17]. Until now, the application research of polarization is still hot. Co-polarization and cross-polarization definition is uniform. Ludwig gave the definition of cross polarization systematically[18].

Ludwig's first definition for cross polarization refers to co-polarization and cross polarization directions that coincide with a rectangular coordinate system. Projection of the electric field vector onto the two Cartesian unit vector $\hat{x}$ and $\hat{y}$ lying in the aperture plane.[18]

$$
\left\{\begin{array}{l}
\hat{u}_{c o}=x \\
\hat{u}_{c r o s s}=y
\end{array}\right.
$$

Ludwig's second definition coincides with the $\theta$ and $\varphi$ directions of a spherical coordinate system. Projection of the electric field vector onto the two spherical unit vector.[18]

$$
\left\{\begin{array}{l}
\hat{u}_{c o}=\hat{\theta} \\
\hat{u}_{\text {cross }}=\hat{\varphi}
\end{array}\right.
$$

Ludwig's third definition is not easy to formulate in terms of simple coordinate system unit vectors. [18]

$$
\left\{\begin{array}{l}
\hat{u}_{c o}=\sin (\varphi) \hat{\theta}+\cos (\varphi) \hat{\varphi} \\
\hat{u}_{c r o s s}=\cos (\varphi) \hat{\theta}-\sin (\varphi) \hat{\varphi}
\end{array}\right.
$$

\section{SIMULATION OF HORN ANTENNA}

\section{A. Linear Polarized Horn}

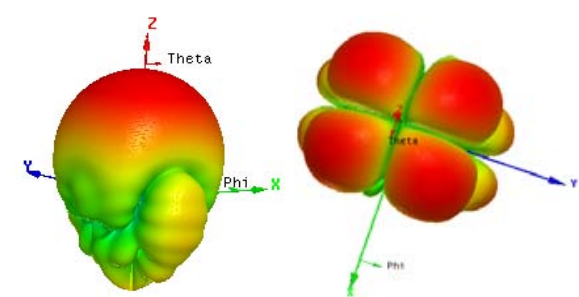

Fig. 1. The sumulation radiation pattern of linera polarized horn antenna. 
The simulation results of the primary and cross polarization radiation pattern of a linear polarized horn antenna are shown in Fig 1. There are four lobes in the cross polarization radiation pattern. And there is a groove of the amplitude between the adjacent two lobes. The position of the main beam of the primary polarization radiation pattern is the same to that of the four lobes of the cross polarization.

Polarization characteristics of $\varphi$ with 45 and 75degrees are shown in Fig. 2. The maximum area in primary polarization is just the center of the four lobes in cross polarization. The cross polarization is gradually reduced from 45 degrees to 75 degrees. So the cross polarization of 75 degrees is lower than that of 45 degrees. And the lowest cross polarization are at 0 degree and 90 degrees, the grooves in Fig. 1.

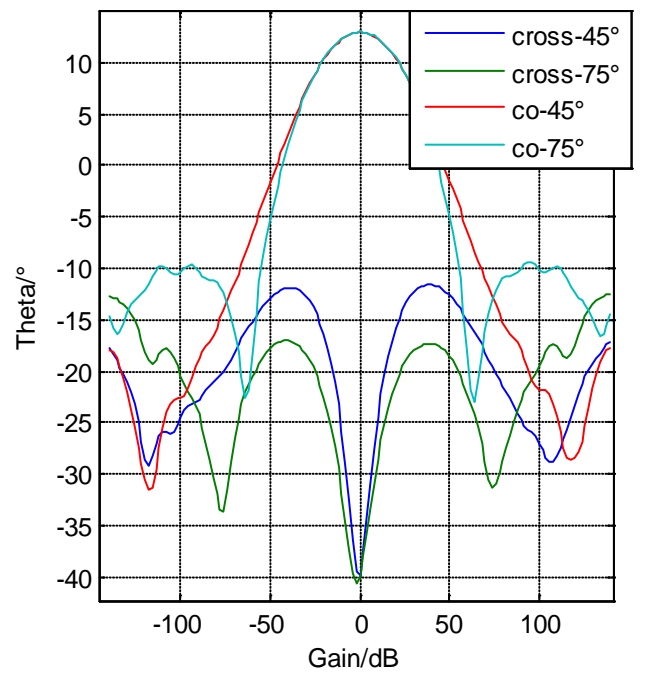

Fig. 2. The polarization characteristics of linear polarized horn antenna.

The phase relationship of co-polarization and cross-polarization is shown in Fig. 3. The phase angle changes with angel of $\varphi$ (called "Phai" in Fig.) when $\theta=40^{\circ}$. It can be seen that the phase fluctuation of co-polarization is very small. But the phase angle in cross-polarization changes very clearly and regularly. The angle is almost the same in each quadrant. There are angle jumps of 180 degrees between any two adjacent quadrants. That means a phase inversion. The phase inversion area is just the amplitude groove in Fig.1.

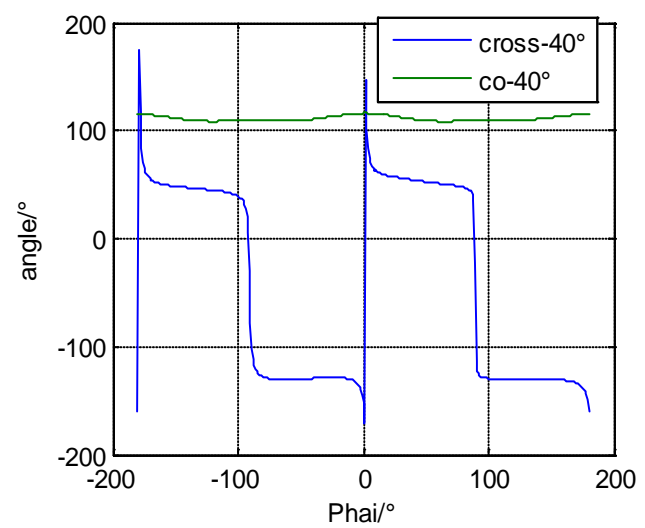

Fig. 3. The co-polarization and cross-polarization phase of linear polarized horn antenna.

\section{B. Circular Polarized Horn}

The simulation results of the cross polarization radiation pattern of a circular polarized horn antenna are shown in Fig 4.

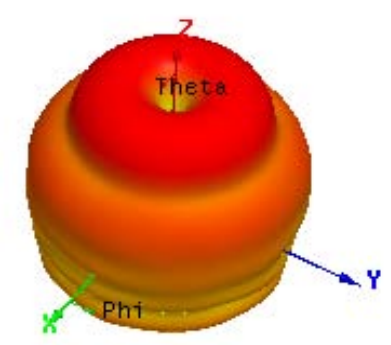

Fig. 4. The sumulation cross-polarization radiation pattern of circular polarized horn antenna.

The cross polarization radiation pattern of circular polarized horn is unlike the linear polarized. There is no 4 lobes here. It is annular with a notch in the center, just like a crater. The location of the notch is corresponding to that of the maximum value in the primary polarization. It can be known from the two-dimensional data. And Fig. 5 is one section of the primary and cross polarization. At any angle of $\varphi$, the value is nearly the same to that in Fig. 5.

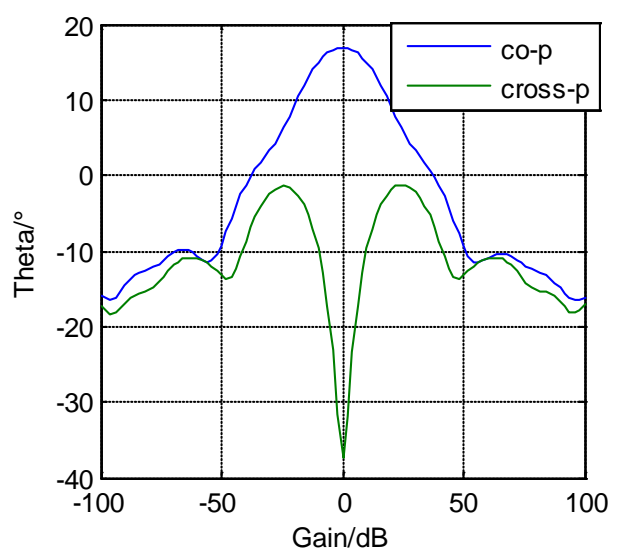

Fig. 5. The polarization characteristics of circular polarized horn antenna. 
The phase relationship of co-polarization and cross-polarization is shown in Fig. 6. The phase angle changes with angel of $\varphi$ (called "Phai" in Fig.6) when $\theta=40^{\circ}$. It can be seen that the phase of both primary and cross polarization varies linearly with the angle of $\varphi$.

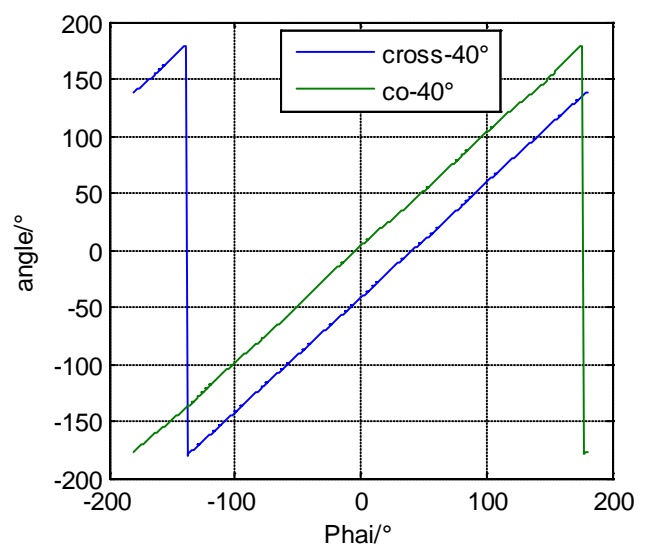

Fig. 6. The co-polarization and cross-polarization phase of circularlinear polarized horn antenna.

\section{MEASUREMENT ANALysis}

One linear polarized horn radiation pattern was measured by the near field measurement method. The primary and cross polarization pattern are shown in Fig. 7. The four lobes can be seen clearly in the cross polarization pattern. In order to obtain the similar results with the simulation, strict coordinate calibration was carried out in the measurement. The polarization definition are the same in both transmitting and receiving system. That means that there is no polarization mismatch between the two systems.

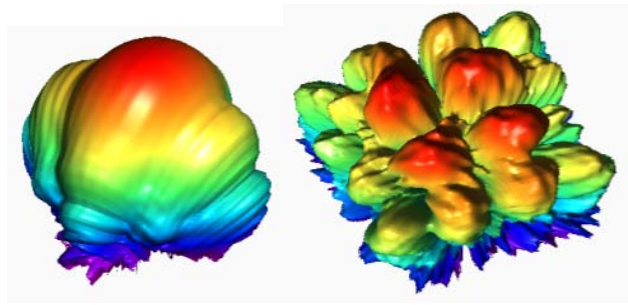

Fig. 7. The measured radiation pattern of linera polarized horn antenna by near field measurement.

Another horn measurement by far field measurement method is shown in Fig. 8 (a). There are two problems here because of the measurement without good coordinate correction. One problem is the maximum value in the primary polarization deviates from the 0 degree of the mechanical coordinate. The other is the shape of cross polarization, without notch, is similar to that of the primary due to the polarization mismatch. Formula * is used here for the polarization matching.

$$
\left\{\begin{array}{l}
u_{p}=u_{p_{-} m} \cdot \cos \phi+u_{c_{-} m} \cdot \sin \phi \\
u_{c}=-u_{p_{-} m} \cdot \sin \phi+u_{c_{-} m} \cdot \cos \phi
\end{array}\right.
$$

Here, $u_{p}$ and $u_{c}$ are the new primary and cross polarization signal voltage after matching. $u_{p_{-} m}$ and $u_{c_{-} m}$ are the primary and cross polarization original measurement data, and $\phi$ is the polarization matching angle. The new result with a notch in the cross polarization is shown in Fig. 8 (b). (a)

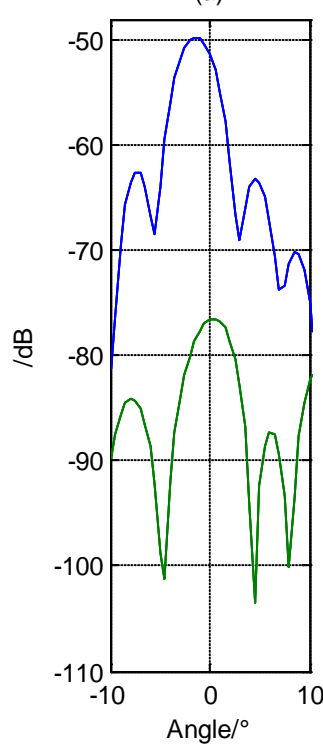

(b)

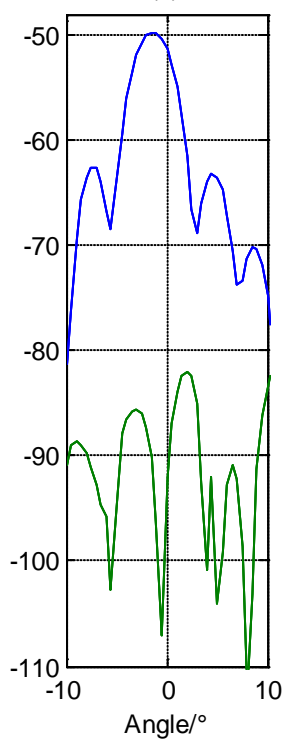

Fig. 8. Fig.8. The measured radiation pattern of linera polarized horn antenna by far field measurement. (a) Original data (b) Data after polarization matching.

\section{CONCLUSION}

The simulation and measurement of horn antenna are carried out for the polarization characteristics analysis. The conclusion is as followings:

- There are four lobes in the cross polarization pattern of linear polarized horn. There are phase inversions between any two adjacent quadrants.

- The cross polarization pattern of circular polarized horn is annular with a notch in the center, but without the 4 lobes. And the phase of both co-polarization and cross-polarization are continuous.

- Strict coordinate correction is need in the measurement to get accurate polarization data.

\section{ACKNOWLEDGMENT}

This work was supported by the Young Scientists Fund of the National Natural Science Foundation of China (Grant No. 61301014) and the Natural Science Foundation of Shandong of China (Grant No. ZR2013FQ017).

\section{REFERENCES}

[1] N.Johnson, M. Civerolo,and N. Lumsden, “Techniques and Methods for Adaptive Single Antenna Radar System Polarization Optimization for Anti Jam and Anti Clutter Applications”, 2015 IEEERadar Conference (RadarCon), 2015, pp. 0210-0213. 
[2] Wei, Z., Ling, W., YingHui, W., Zhaolin, Z., and Meng, Z., “Joint Polarized and Spatial Domain Anti-Jamming Method for Gnss”, 2013 IEEE International Conference on Signal Processing, Communication and Computing (ICSPCC), 2013, pp. 1-5.

[3] Xu, Z., Xiong, Z., and Xiao, S., “A Novel Alternate Polarization Array and Its Filtering Performance”, 2011 IEEE CIE International Conference onRadar , 2011, pp. 1890-1892.

[4] S.Li, Z.Wang, S.Lou, and Y.Zhan, "Gps Anti-Jamming Utilizing Dual-Polarized Antenna”, Cross Strait Quad-Regional Radio Science and Wireless Technology Conference (CSQRWC), 2011, pp.502-506.

[5] Y.Chang, Y.Li, X.Wang, and S.Xiao, "New Target Detection Method in Strong Active Jamming Background for Polarimetric Radar”, 2010 IEEE 10th International Conference onSignal Processing (ICSP), 2010, pp.1947-1950.

[6] L.-Z.Song, X.-l.Qiao, S.-f.Fu, and Q.Wu, “A Kind of Signal Processing Method for the Polarization Phased Array Radar”, 5th International Conference onWireless Communications, Networking and Mobile Computing, 2009, pp. 1-4.

[7] E.Markin, 'Jamming Detection in Providing for Radar Jamming Immunity', IEEE EUROCON '09, 2009, pp.1565-1567.

[8] T.Jin, X.Qi, X.Qiao, M.Zhang, S.Yuan, and Q.Zhang, 'Anti-Full Polarization Active Jamming', 2nd IEEE Conference on Industrial Electronics and Applications, 2007, pp. 2718-2722.

[9] Lizhong, S., Xiaolin, Q., Xiande, M., and Ming, J., "Study on the Method of Polarization Suppression of Cheating Jamming in Pulse Doppler Radar," Journal ofSystems Engineering and Electronics, vol. 16 pp. 310-315, 2005.

[10] D.Giuli, "Polarization Diversity in Radars," Proceedings of the IEEE, vol. 74, 1986, pp. 245-269.
[11] E. Lier, and S.A.Skyttemyr, “A Compact Shaped Single-Reflector Offset Antenna with Low Cross-Polarization”, Antennas and Propagation Society International Symposium, 1991, pp. 682-689.

[12] A.K.Bhattacharyya, and G.Goyette, "A Novel Horn Radiator with High Aperture Efficiency and Low Cross-Polarization and Applications in Arrays and Multibeam Reflector Antennas," IEEE Transactions onAntennas and Propagation, , 2004,vol. 52, pp. 2850-2859.

[13] Lei, W., Xiaoxing, Y., Shunli, L., Hongxin, Z., Leilei, L., and Ming, Z., "Phase Corrected Substrate Integrated Waveguide H-Plane Horn Antenna with Embedded Metal-Via Arrays," IEEE Transactions onAntennas and Propagation, 2014,vol. 62, pp. 1854-1861.

[14] Othman, M.A., Aziz, M.Z.A.A., Saysoo, N., and Othman, A.R., "Development of Ultra-Wideband (Uwb) Horn Antenna Using Approximation Method”, 2012 IEEE Symposium on Wireless Technology and Applications (ISWTA), 2012, pp. 276-279.

[15] Young-Bae, J., Soon-Young, E., Soon-Ik, J., and Chang-Joo, K., “Novel Ka-Band Microstrip Antenna Fed Circular Polarized Horn Array Antenna”, IEEE Antennas and Propagation Society International Symposium 2004, 2004, pp. 2476-2479.

[16] Di, W., Zhenghe, F., and Wei, L., “A Novel Conical Horn Antenna Loaded with Ball Cone Dielectric”, 2014 3rd Asia-Pacific Conference onAntennas and Propagation (APCAP), 2014, pp. 631-634.

[17] M.M.A.El Futuhand S.I.Ghobril, "The Effect of Deviation of Feed Polarization Characteristics from That of a Huygens Source on Cross-Polarization in Reflector Antennas," Radio and Electronic Engineer, 1974,vol. 44, pp. 269-272.

[18] A. Ludwig, "The Definition of Cross Polarization," IEEE Transactions onAntennas and Propagation, 1973, vol. 21, pp. 116-119. 